\title{
FATORES DE RISCO, NÍVEIS DE ESPIRITUALIDADE E USO DE ÁLCOOL EM ESTUDANTES DE DOIS CURSOS DE ENFERMAGEM*
}

\section{Sandra Cristina Pillon ${ }^{1}$; Manoel Antônio dos Santos ${ }^{2}$; Angélica Martins de Souza Gonçalves ${ }^{3}$; Keila Maria de Araújo ${ }^{4}$; Anderson Funai ${ }^{5}$}

Este é um estudo transversal e teve por objetivo analisar as relações entre fatores de risco, níveis de espiritualidade e uso de álcool em estudantes de dois cursos de enfermagem. A amostra foi composta por 313 estudantes. Foram utilizados: questionário de informações sociodemográficas, Teste de Identificação do Uso do Álcool e Escala de Espiritualidade. Houve predomínio de jovens do sexo feminino, solteiros e católicos. O uso de álcool esteve presente em $83,4 \%$ da amostra e quase um terço o consumia em níveis problemáticos. Os fatores de risco foram sexo masculino e baixa pontuação nos níveis de espiritualidade. Estudos dessa natureza são de grande valia quando da elaboração de programas de prevenção ao uso de álcool, no âmbito universitário.

Descritores: Uso de álcool; Fator de Risco; Estudante de Enfermagem; Prevenção.

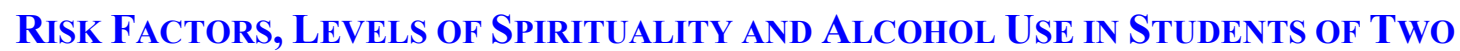 NURSING COURSES ${ }^{\dagger}$}

The purpose of this cross-sectional study was to analyze the relationships existing between risk factors, levels of spirituality and alcohol use among students of two nursing courses. The sample consisted of 313 students. The following were used: questionnaire with sociodemographic information, Alcohol Use Disorder Identification Tests, and Spirituality Scale. Most participants were young females, single and catholic. Alcohol use was present in $83.4 \%$ of the sample and in nearly one third it was considered to be an alcohol use disorder. The risk factors were male gender and a low score on the spirituality levels. Studies of this nature are of great value in planning programs for the prevention of alcohol use in the university.

Descriptors: Alcohol Use; Risk Factor; Nursing Student; Prevention.

\footnotetext{
*Trabalho agraciado com o primeiro lugar (Prêmio: Maria Aparecida Minzoni), no X Encontro de Pesquisadores em Saúde Mental e Enfermagem Psiquiátrica. Ribeirão Preto, 2008.

${ }^{\dagger}$ This study was first-prize winner (Prize: Maria Aparecida Minzoni) in the $10^{\text {th }}$ Meeting of Researchers in Mental Health and Psychiatric Nursing. Ribeirão Preto, 2008.

1-Enfermeira, Livre Docente, Professor Associado, Escola de Enfermagem de Ribeirão Preto, Universidade de São Paulo, Centro Colaborador da OMS para o Desenvolvimento da Pesquisa em Enfermagem, SP, Brasil. Email: pillon@eerp.usp.br

2-Psicólogo, Doutor em Psicologia, Professor Doutor, Faculdade de Filosofia, Ciências e Letras de Ribeirão Preto, Universidade de São Paulo, SP, Brasil.E-mail: masantos@ffclrp.usp.br

3-Enfermeira, Doutoranda em Enfermagem Psiquiátrica, Escola de Enfermagem de Ribeirão Preto, Universidade de São Paulo, Centro Colaborador da OMS para o Desenvolvimento da Pesquisa em Enfermagem, SP, Brasil. Email: angelicamartins@usp.br

4-Enfermeira, Professor, Pontifícia Universidade Católica, Poços de Caldas, MG, Brasil. E-mail: kmarausp@yahoo.com.br

5-Enfermeiro, Mestre em Enfermagem, Professor Assistente, Universidade Federal do Recôncavo da Bahia, BA, Brasil. E-mail: afunai@,famema.br
}

Autor Correspondente: Angélica Martins de Souza Gonçalves Endereço para Correspondência: Universidade de São Paulo, Escola de Enfermagem de Ribeirão Preto, Departamento de Enfermagem Psiquiátrica e Ciência Humanas, Avenida Bandeirantes, 3900, Bairro Monte Alegre, CEP: 14040-902, Ribeirão Preto, SP, Brasil. E-mail: angelicamartins@usp.br 


\section{FACTORES DE RIESGO, NIVELES DE ESPIRITUALIDAD Y USO DE ALCOHOL EN ESTUDIANTES DE DOS CURSOS DE ENFERMERÍA}

Estudio transversal, tuvo por objetivo analizar las relaciones entre factores de riesgo, niveles de espiritualidad y uso de alcohol en estudiantes de dos cursos de enfermería. La muestra se compuso de 313 estudiantes. Fueron utilizados: cuestionario de informaciones sociodemográficas, Test de Identificación del Uso de Alcohol y Escala de Espiritualidad. Existió predominio de jóvenes de sexo femenino, solteros y católicos. El uso de alcohol se hizo presente en $83,4 \%$ de la muestra, casi un tercio consumía en niveles problemáticos. Los factores de riesgo fueron: sexo masculino y baja puntuación en niveles de espiritualidad. Estudios de tal naturaleza resultan altamente valorables en la elaboración de programas de prevención del uso del alcohol en el ámbito universitario.

Descriptores: Uso del Alcohol; Factor de Riesgo; Estudiante de Enfermeria; Prevención. 


\section{Introdução}

Evidências apontam que o uso de álcool e drogas ilícitas está aumentando entre os jovens e que o público universitário tem sido o grande alvo desse consumo. Os estudantes tendem a beber em níveis prejudiciais à saúde e, ainda, experimentar drogas ilícitas ${ }^{(1)}$. Diversos grupos de estudantes pouco diferenciam entre si, no que diz respeito ao padrão de consumo. Além disso, os estudantes da área de saúde, supostamente, têm acesso a conhecimentos sobre os potenciais prejuízos causados pelo álcool.

Dentre os diversos fatores associados ao consumo de álcool, por estudantes universitários da área de ciências biológicas (incluindo os de Enfermagem), renda familiar elevada e ausência de religião foram considerados aqueles de maior risco, não somente para o álcool, mas também para o tabaco e as drogas ilícitas ${ }^{(2)}$. Os problemas relacionados ao consumo de álcool por estudantes universitários representam motivo de preocupação e resultam em demandas por melhores cuidados de saúde, educação em saúde e sistemas de apoio nas universidades ${ }^{(1-4)}$. Se os estudantes de Enfermagem são vulneráveis ao abuso de substâncias psicoativas, esses problemas podem ser identificados logo no início de sua formação, de modo que intervenções oportunas possam reduzir os riscos de prejuízos futuros ${ }^{(5)}$.

Estudos com população universitária foram desenvolvidos em diversas cidades brasileiras com o objetivo de demonstrar não apenas o padrão de consumo, mas suas consequências devido ao uso abusivo de bebidas.

Estudos realizados com diversos 
grupos de estudantes universitários, utilizando o Teste de Identificação dos Problemas Relacionados ao Uso do Álcool (AUDIT), identificaram o uso problemático de álcool (pontuação $\geq 8$ ). Os índices encontrados variam de acordo com o estudo: $20,5 \%{ }^{(3)}, \quad 21,36 \%{ }^{(6)}$, $25,71 \%^{(7)}, 26 \%{ }^{(8)}$ e $44 \%{ }^{(9)}$.

Um conceito que parece desempenhar papel significativo no consumo de álcool, em estudantes universitários, é a espiritualidade. Embora haja poucos estudos sobre esse tema, algumas pesquisas foram desenvolvidas utilizando esse constructo e as possíveis relações com o uso de substâncias psicoativas. No entanto, nota-se que várias investigações são de natureza epidemiológica e descritiva, interessadas apenas na classificação de padrões de consumo ${ }^{(10-}$ 12).

Dentre os resultados encontrados nas pesquisas com estudantes universitários $^{(3,10,12)}, \quad$ a religião $\quad \mathrm{e} \quad \mathrm{a}$ espiritualidade se destacaram como fatores de proteção em relação ao uso problemático de álcool em estudantes. Os autores identificaram porcentagem notável de estudantes que não declararam a afiliação religiosa dentre os que faziam consumo problemático de bebidas alcoólicas. Uma particularidade observada nos estudos relaciona-se ao tipo de afiliação religiosa. Os estudantes católicos e espíritas apresentaram consumo problemático de bebidas alcoólicas maior quando comparado aos evangélicos.

Entre os estudos que mencionam as possíveis relações entre espiritualidade e o uso de drogas, estudo realizado na Irlanda, envolvendo 458 estudantes universitários, mostrou maior consumo de álcool entre os estudantes com menor 
crença em Deus e menor frequência aos cultos religiosos $^{(13)}$.

A religiosidade/espiritualidade tornou-se tema de suma relevância a ser explorado, de modo que foi enfatizada e considerada como variável que influencia a saúde mental e que está sendo negligenciada pela psiquiatria nos seus estudos e programas de tratamento e prevenção $^{(14)}$. Nas pesquisas realizadas na população geral, especificamente com estudantes universitários, essa variável tem sido explorada apenas como uma das informações sociodemográficas, o que dificulta compreensão mais ampliada do modo como influencia os indivíduos, podendo funcionar como fator protetor frente ao uso de álcool.

Nesse contexto, vários estudos têm identificado a importância da espiritualidade na vida pessoal, nas relações sociais, nas atitudes e representações, relacionadas ao processo saúde/doença, assim como na composição dos sintomas psiquiátricos, incluindo o uso de substâncias psicoativas ${ }^{(15)}$.

As dimensões religiosas relacionamse à modulação do uso de álcool e drogas. Ao vincular-se a uma religião e se envolver com os padrões de religiosidade, a pessoa adere a um conjunto de valores, símbolos, comportamentos e práticas sociais, na qual a aceitação ou recusa do uso de álcool e/ou outras drogas está inserida $^{(16)}$.

Considerada como fator de proteção em relação ao uso de álcool e outras drogas, a prática religiosa tem sido objeto de estudo de vários pesquisadores. A literatura evidência ênfase na compreensão dos fatores de risco, atribuindo pouca atenção aos fatores protetores que podem ser de grande valia 
na elaboração de programas de prevenção ao uso de drogas ${ }^{(10-11,16-19)}$.

Estudantes universitários constituem grupo vulnerável em relação ao consumo de bebidas alcoólicas ${ }^{(3-4,7-9)}$. Esses jovens estão vivenciando um período de transição no ciclo vital, o que os torna mais propensos ao uso de álcool. Essa vulnerabilidade inerente ao processo de amadurecimento pode ser agravada por fatores tais como: a saída da casa dos pais, a exposição ao ambiente universitário que, muitas vezes, é permissivo ao uso de drogas, tornando quase que obrigatório embebedar-se em circunstâncias festivas, além dos modos de enfrentamento das dificuldades do dia a dia ${ }^{(3)}$.

Mediante o exposto, a relevância do tema e a escassez de estudos que avaliam a espiritualidade e o uso de álcool em estudantes de enfermagem, no Brasil,

justificam o desenvolvimento do presente estudo. $\mathrm{O}$ presente estudo foi desenvolvido com base na experiência prática dos pesquisadores como docentes de cursos de Enfermagem, que têm mostrado, cada vez mais, que estudantes com problemas, relacionados ao uso de substâncias psicoativas, principalmente o álcool, estão batendo à porta em busca de ajuda.

Este estudo teve por objetivo analisar as relações entre fatores de risco, níveis de espiritualidade e uso de álcool em estudantes de dois cursos de enfermagem.

\section{Método}

O desenho metodológico do
presente estudo é do tipo descritivo
exploratório, de coorte transversal. O
estudo foi desenvolvido em duas escolas


de Enfermagem, localizadas no interior dos Estados de São Paulo e Minas Gerais. A amostra foi composta por $313(100 \%)$ estudantes do primeiro ao quarto ano de graduação em Enfermagem, sendo 122 (39\%) oriundos da Escola A e 191 (61\%) da Escola B. A pesquisa foi desenvolvida nos anos 2008 e 2009.

Para a coleta de dados foram utilizados os seguintes instrumentos: questionário contendo informações sociodemográficas, elaborado para o presente estudo, Teste de Identificação do Uso do Álcool (AUDIT) ${ }^{(20)}$ e Escala de Espiritualidade (Spirituality Self-Rating Scale-SSRS) ${ }^{(18)}$.

O AUDIT é instrumento de rastreamento do uso do álcool, validado para o contexto brasileiro. Trata-se de teste de autopreenchimento, contendo 10 perguntas que avaliam o padrão de consumo do álcool, os sinais e sintomas da dependência e os problemas decorrentes do uso do álcool. Para a apuração dos dados, soma-se a pontuação obtida nas respostas que varia de zero a 40 pontos e classifica em duas categorias: sujeitos com pontuação de 0 a 7 (AUDIT $\leq 8$ ), que correspondem aos abstinentes ou bebedores, dentro dos limites recomendados pela Organização Mundial de Saúde, e, acima de 8 pontos (AUDIT $\geq 8$ ), caracteriza as pessoas que consomem bebidas alcoólicas em níveis problemáticos $^{(20)}$.

A SSRS é escala que contém seis itens que refletem a orientação para espiritualidade do indivíduo, ou seja, se a pessoa considera ou julga mais ou menos importante as questões relativas às dimensões espirituais/religiosas e como as aplica em sua vida. Foi elaborada levando em consideração alguns itens sobre práticas religiosas e preceitos 
teóricos dos Doze Passos dos Alcoólatras

Anônimos. Alguns desses preceitos, que não estão relacionados à doutrina religiosa, se referem à crença de que um Poder Superior teria potencial na recuperação, necessidade de reconhecimento de falhas pessoais e de prática espiritual $^{(18)}$.

As respostas da SSRS são do tipo Likert, variando de 1 - discordo muito a 5 - concordo muito. A soma dos itens permite a leitura dos escores, ou seja, quanto maior o escore maiores os níveis de orientação espiritual. Os itens da escala referem-se à intervenção divina no cotidiano e prática de rituais religiosos, como rezar. Essa escala, que avalia os níveis de espiritualidade foi validada no Brasil $^{(19)}$. O teste de confiabilidade da escala, envolvendo as duas escolas, apresentou coeficiente alpha de Cronbach igual a 0,86 , valor considerado aceitável, o que valida sua utilização no contexto brasileiro $^{(19)}$.

\section{Procedimento}

Autorizações formais foram concedidas pela direção das respectivas escolas. A coleta de dados ocorreu em sala de aula, mediante agendamento prévio pela coordenação de ensino, para que não interferisse nas atividades acadêmicas. Os estudantes que aceitaram participar do estudo firmaram o termo de consentimento livre e esclarecido, após terem recebido orientações quanto ao objetivo da pesquisa e garantia do anonimato. A participação dos estudantes foi voluntária com total liberdade de recusarem a colaborar com a investigação.

Uma caixa, simulando uma urna, 
foi colocada sobre a mesa da sala de aula para que os questionários fossem ali depositados após o término do preenchimento. Mesmo que os alunos optassem por deixá-los em branco, deveriam seguir as mesmas regras para que não fossem identificados. Após a entrega do último questionário, o aplicador realizou uma checagem para conferir o número de alunos matriculados da sala em questão, a fim de avaliar os faltosos e, posteriormente, realizar uma nova visita à sala de aula para tentar recrutá-los.

Elaborou-se um banco de dados no programa Statistical Package for Social Science version 11 for Windows. A análise dos dados ocorreu mediante o uso da estatística descritiva (frequência, porcentagem, média e desvio padrão). Para a análise de associações utilizou-se o teste de qui-quadrado. Adotou-se intervalo de confiança de 95\%.

$$
\mathrm{O} \text { projeto foi aprovado pelo }
$$

Comitê de Ética em Pesquisa, Processo ${ }^{\circ}$ 0924/2008.

\section{Resultados}

No que concerne às características sociodemográficas da amostra, houve predomínio do sexo feminino - 257 (82,1\%), média de idade de $23,4 \pm 5,52$ anos, variando de 18 a 48 anos. Em relação ao estado civil, 248 (79,2\%) eram solteiros, $65(20,8 \%)$ casados ou em união consensual. Quanto à religião, 206 $(65,8 \%)$ autodeclararam-se católicos, 61 (19,5\%) evangélicos, 26 (8,3\%) espíritas, 17 (5,4\%) não possuíam religião e $3(1 \%)$ outras. No que diz respeito à distribuição do ano no qual os estudantes estavam cursando, $78(24,9 \%)$ pertenciam ao 
primeiro ano, $100(31,9 \%)$ ao segundo ano, $88(27,5 \%)$ ao terceiro ano e 49 $(15,7 \%)$ ao quarto ano.

Em relação ao consumo de bebidas alcoólicas, $261 \quad(83,4 \%)$ consumiram no último ano. Do total de estudantes, $230 \quad(73,4 \%) \quad$ pontuaram AUDIT $\leq 8$ e $83(26,6 \%)$ AUDIT $\geq 8$.
Embora haja porcentagens consideráveis de estudantes, de ambas as escolas, que consumiam em baixos níveis de risco, destacou-se o beber problemático em alunos de primeiro ao terceiro ano da escola B e do quarto ano da escola A, conforme mostra a Tabela 1.

Tabela 1 - Distribuição dos estudantes de Enfermagem, segundo o ano de graduação, a classificação obtida no AUDIT e a escola de origem $(\mathrm{N}=313)$

\begin{tabular}{|c|c|c|c|c|c|c|c|c|c|c|c|}
\hline \multirow{2}{*}{ AUDIT } & & \multicolumn{2}{|c|}{$1^{\circ}$ ano } & \multicolumn{2}{|c|}{$2^{\circ}$ ano } & \multicolumn{2}{|c|}{$3^{\circ}$ ano } & \multicolumn{2}{|c|}{$4^{\circ}$ ano } & \multicolumn{2}{|c|}{ Total } \\
\hline & & $\mathbf{N}$ & $\%$ & $\mathbf{N}$ & $\%$ & $\mathbf{N}$ & $\%$ & $\mathbf{N}$ & $\%$ & $\mathbf{N}$ & $\%$ \\
\hline \multirow{3}{*}{$\leq 8$} & Escola A & 25 & 41,7 & 29 & 44,6 & 27 & 39,7 & 16 & 43,2 & 97 & 42,2 \\
\hline & Escola B & 35 & 58,3 & 36 & 55,4 & 41 & 60,3 & 21 & 56,8 & 133 & 57,8 \\
\hline & Total & 60 & 100 & 65 & 100 & 68 & 100 & 37 & 100 & 230 & 100 \\
\hline \multirow{3}{*}{$\geq 8$} & Escola A & 6 & 33,3 & 6 & 17,1 & 5 & 27,8 & 8 & $66,7^{*}$ & 25 & 30,1 \\
\hline & Escola B & 12 & $66,7^{*}$ & 29 & $82,9^{*}$ & 13 & $72,2^{*}$ & 4 & 33,3 & 58 & 69,9 \\
\hline & Total & 18 & 100 & 35 & 100 & 18 & 100 & 12 & 100 & 83 & 100 \\
\hline
\end{tabular}

Ao avaliar as médias de idade, os alunos da Escola A eram mais jovens $(20,95 \pm 1,94$ anos, variando de 17 a 28 anos, quando comparados aos estudantes

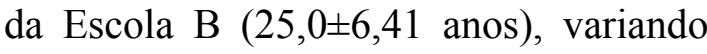
de 17 a 48 anos. Houve diferença estatisticamente significativa entre as médias de idade das escolas A e B 
$(\mathrm{p}<0,05)$.

Os estudantes de enfermagem, de

ambas as

escolas,

eram

predominantemente do sexo feminino

(82,1\% da amostra) e pontuaram AUDIT $\leq 8$. Em contrapartida, identificou-se o beber problemático no sexo masculino (AUDIT $\geq 8$ ), conforme mostra a Tabela

Tabela 2 - Distribuição dos estudantes de Enfermagem, segundo o sexo, a classificação obtida AUDIT e a escola de origem $(\mathrm{N}=313)$

\begin{tabular}{|c|c|c|c|c|c|c|c|}
\hline \multirow{2}{*}{ AUDIT } & & \multicolumn{2}{|c|}{ Feminino } & \multicolumn{2}{|c|}{ Masculino } & \multicolumn{2}{|c|}{ Total } \\
\hline & & $\mathbf{N}$ & $\%$ & $\mathbf{N}$ & $\%$ & $\mathbf{N}$ & $\%$ \\
\hline \multirow{3}{*}{$\leq 8$} & Escola A & 92 & 45,3 & 5 & 18,5 & 97 & 42,2 \\
\hline & Escola B & 111 & 54,7 & 22 & 81,5 & 133 & 57,8 \\
\hline & Total & 203 & 100 & 27 & 100 & 230 & 100 \\
\hline \multirow{3}{*}{$\geq 8$} & Escola A & 21 & 38,9 & 4 & 13,8 & 25 & 30,1 \\
\hline & Escola B & 33 & 61,1 & 25 & 86,2 & 58 & 69,9 \\
\hline & Total & 54 & 100 & 29 & 100 & 83 & 100 \\
\hline
\end{tabular}

Em relação ao estado civil, pontuação no AUDIT $\geq 8$, conforme identificou-se associação positiva entre os mostra a Tabela 3. estudantes casados da escola B e a

Tabela 3 - Distribuição dos estudantes de Enfermagem, segundo o estado civil, a classificação obtida no AUDIT e a escola de origem $(\mathrm{N}=313)$

\begin{tabular}{|c|c|c|c|c|c|c|c|}
\hline \multirow{2}{*}{ AUDIT } & & \multicolumn{2}{|c|}{ Solteiro } & \multicolumn{2}{|c|}{ Casado } & \multicolumn{2}{|c|}{ Total } \\
\hline & & $\mathbf{N}$ & $\%$ & $\mathbf{N}$ & $\%$ & $\mathbf{N}$ & $\%$ \\
\hline \multirow{3}{*}{$\leq 8$} & Escola A & 94 & 51,4 & 3 & 6,4 & 97 & 42,2 \\
\hline & Escola B & 89 & 48,6 & 44 & 93,6 & 133 & 57,8 \\
\hline & Total & 183 & 100 & 47 & 100 & 230 & 100 \\
\hline$\geq 8$ & Escola A & 25 & 37,9 & - & - & 25 & 30,1 \\
\hline
\end{tabular}




\begin{tabular}{ccccccc}
\hline Escola B & 41 & 62,1 & 17 & 100 & 58 & 69,9 \\
Total & 66 & 100 & 17 & 100 & 83 & 100 \\
\hline
\end{tabular}

Quanto aos níveis de estudantes da escola B pontuaram baixos espiritualidade, os estudantes da escola A, níveis de espiritualidade e AUDIT $\geq 8$, classificados AUDIT $\leq 8$, pontuaram conforme demonstrado na Tabela 4 . baixos níveis de espiritualidade. Os

Tabela 4 - Distribuição dos estudantes de Enfermagem, segundo a pontuação obtida na SRSS e no AUDIT e a escola de origem $(\mathrm{N}=313)$

\begin{tabular}{|c|c|c|c|c|c|c|c|}
\hline \multirow{3}{*}{ AUDIT } & & \multicolumn{4}{|c|}{ SRSS } & \multirow{2}{*}{\multicolumn{2}{|c|}{ Total }} \\
\hline & & \multicolumn{2}{|c|}{$\leq 15$} & \multicolumn{2}{|c|}{$\geq 15$} & & \\
\hline & & $\mathbf{N}$ & $\%$ & $\mathbf{N}$ & $\%$ & $\mathbf{N}$ & $\%$ \\
\hline \multirow{3}{*}{$\leq 8$} & Escola A & 14 & 12,0 & 83 & 74,1 & 97 & 42,4 \\
\hline & Escola B & 103 & 88,0 & 29 & 25,9 & 132 & 57,6 \\
\hline & Total & 117 & 100 & 112 & 100 & 229 & 100 \\
\hline \multirow{3}{*}{$\geq 8$} & Escola A & 3 & 6,4 & 22 & 61,1 & 25 & 30,1 \\
\hline & Escola B & 44 & 93,6 & 14 & 38,9 & 58 & 69,9 \\
\hline & Total & 47 & 100 & 36 & 100 & 83 & 100 \\
\hline
\end{tabular}

$\mathrm{Na}$ amostra, identificou-se pontuaram AUDIT $\geq 8$, a média foi de homogeneidade em relação à idade e ao $\quad 23,29 \pm 4,68$ anos. Não houve associação uso do álcool. O grupo com pontuação no estatisticamente significativa $(\mathrm{p}>0,05)$.

AUDIT $\leq 8$ apresentou média de idade de Ao avaliar a pontuação da SRSS, $23,49 \pm 5,8$ anos e, para os estudantes que identificou-se que os estudantes da escola 
A apresentaram média maior $(21,0 \pm 4,87$, variando de 10 a 30 pontos), quando comparados aos da escola B $(12,9 \pm 4,0$, variando de 6 a 29 pontos). As diferenças foram estatisticamente significativas $(\mathrm{p}<0,05)$.

A respeito da afiliação religiosa, de acordo com a classificação do AUDIT e da escala de espiritualidade, houve diferenças significativas. Não ter nenhuma afiliação religiosa ou ser católico foi associado a menor nível de espiritualidade e AUDIT $>8$. Os estudantes espíritas pontuaram AUDIT $<8$ e AUDIT $>8$ e altos índices na escala de espiritualidade. Os estudantes evangélicos pontuaram AUDIT $<8$ e apresentaram baixo nível de espiritualidade, conforme mostra a Tabela 5.

Tabela 5 - Distribuição dos estudantes de Enfermagem, segundo afiliação religiosa, classificação obtida no AUDIT e na SRSS $(\mathrm{N}=313)$

\begin{tabular}{|c|c|c|c|c|c|c|c|c|c|c|c|}
\hline \multirow{2}{*}{ AUDIT } & & \multicolumn{2}{|c|}{ Nenhuma } & \multicolumn{2}{|c|}{ Católico } & \multicolumn{2}{|c|}{ Evangélico } & \multicolumn{2}{|c|}{ Espírita } & \multicolumn{2}{|c|}{ Total } \\
\hline & & $\mathbf{N}$ & $\%$ & $\mathbf{N}$ & $\%$ & $\mathbf{N}$ & $\%$ & $\mathbf{N}$ & $\%$ & $\mathbf{N}$ & $\%$ \\
\hline \multirow{3}{*}{$<8$} & SRSS $<15$ & 7 & 50,0 & 74 & 49,7 & 30 & 61,2 & 6 & 35,3 & 117 & 51,1 \\
\hline & SRSS $>15$ & 7 & 50,0 & 75 & 50,3 & 19 & 38,8 & 11 & 64,7 & 112 & 48,9 \\
\hline & Total & 14 & 100 & 149 & 100 & 49 & 100 & 17 & 100 & 229 & 100 \\
\hline \multirow{3}{*}{$>8$} & SRSS $<15$ & 4 & 80,0 & 29 & 60,4 & 61,9 & 13 & 1 & 11,1 & 47 & 56,6 \\
\hline & SRSS $>15$ & 1 & 20,0 & 19 & 39,6 & 8 & 38,1 & 8 & 88,9 & 36 & 43,4 \\
\hline & Total & 5 & 100 & 48 & 100 & 21 & 100 & 9 & 100 & 83 & 100 \\
\hline
\end{tabular}

\section{Discussão}

A questão do uso do álcool em estudantes tem se tornado um desafio no 
âmbito universitário, uma vez que a bebida alcoólica permeia o cotidiano desses jovens e constituem importante estímulo da sociabilidade na vida universitária. A literatura sobre esse tema é ampla, complexa e preocupante, uma vez que o beber em níveis problemáticos resulta em consequências negativas, não se restringindo apenas à vida universitária dos estudantes que fazem consumo de bebidas alcoólicas. Dados internacionais estimam que, anualmente, $\quad 1.400$ estudantes universitários, com idade de 18 a 24 anos, morreram como conseqüência de danos causados pelo uso do álcool e 500.000 estiveram envolvidos em prejuízos não intencionais, quando intoxicados pelo álcool $^{(21)}$.

No presente estudo, identificou-se que $\quad 83,4 \%$ estudantes consumiram bebidas alcoólicas no último ano, dentre os quais $26,6 \%$ estudantes consumiram em níveis problemáticos. Os dados corroboram aqueles apresentados na literatura nacional ${ }^{(3,6,8)}$, sendo que o uso problemático de álcool em estudantes de Enfermagem alcançam índices de $20,5 \%^{(3)}, \quad 21,3 \%^{(6)}$ e $25,71 \%^{(7)} ; \quad$ em estudantes da área de saúde esse índice é de $26 \%^{(8)}$. Frente a esses índices de consumo, percebe-se que o uso do álcool está muito presente na vida dos universitários.

O beber problemático do álcool apresentou-se associado aos estudantes do primeiro ao terceiro ano da escola $\mathrm{B}$, enquanto que, na escola $\mathrm{A}$, apenas os estudantes do quarto ano consumiam desse modo (Tabela 1). Embora se reconheçam algumas limitações no presente estudo, frente às peculiaridades de cada escola, e a forma como ocorre a socialização desses alunos, há de se repensar o consumo de bebidas alcoólicas 
dos ingressantes, bem como durante a graduação, considerando que os alunos do quarto ano estão fazendo uso de bebidas alcoólicas em níveis problemáticos.

A esse respeito, a literatura menciona que o consumo de drogas em estudantes está relacionado à vida social mais intensa, talvez porque, fora de casa ou em grupos, o acesso às drogas seja mais fácil, ou, então, porque esses indivíduos são, devido à educação que receberam ou ao meio onde estão inseridos, mais "abertos" e com menos “tabus" em relação a esse consumo ${ }^{(22)}$.

Dentre as principais características da amostra em relação ao beber, identificou-se que o maior consumo de bebidas alcoólicas esteve presente entre o sexo masculino (Tabela 2), embora a amostra do presente estudo seja composta predominantemente por estudantes do sexo feminino. Esses dados corroboram aqueles apresentados na literatura nacional $^{(3,6)}$, uma vez que o padrão de consumo do álcool difere em relação ao gênero e que os homens jovens são os maiores consumidores, em frequência e quantidade, de bebidas alcoólicas, quando comparados às mulheres ${ }^{(3)}$.

O uso problemático de álcool também se apresentou associado ao estado civil. Os solteiros apresentaram maiores porcentagens. Sobre esse respeito, a literatura é muito controversa, mas, de modo geral, ser casado tem sido considerado como fator de proteção ao uso de álcool ${ }^{(3)}$.

Baixos níveis na escala de espiritualidade foram observados em ambos os grupos de consumo de álcool, na escola B. Com base nos resultados encontrados na presente pesquisa, pode-se supor que a espiritualidade não está atuando de forma protetora como se 
esperava frente ao uso de álcool para um dos grupos de estudantes da escola B, o que não ocorreu com os grupos da escola A. Esses achados estão em consonância com a literatura que sugere a espiritualidade, de modo geral, embora, nem sempre, demonstre ter efeito protetor sobre o uso de álcool e outras drogas, entre adolescentes e estudantes universitários $^{(5)}$.

Não houve diferenças que associam o consumo problemático do álcool e a idade, em ambas as escolas. Esse dado chama a atenção uma vez que a literatura considera que a idade, principalmente no período da adolescência, caracteriza um dos potenciais fatores de risco para o consumo do álcool e de outras drogas.

Nesse sentido, embora haja diferenças culturais, de sexo, idade, entre outras, os jovens na faixa dos 20 anos compõem parte da população que bebe muito, embriaga-se com regularidade e registra problemas com bebida, frequentemente relacionados a esse padrão de consumo. O beber nessa faixa de idade também reflete o estilo de vida, a exuberância e irresponsabilidade da juventude, e grande parte dos comportamentos, que na época pareciam indicar doença de ingestão, terá, anos mais tarde, modificado muito sua apresentação ou ter mesmo desaparecido. O traço mais notável desse comportamento é a sua fluidez, porém, os perigos associados ao beber problemático na juventude não devem ser ignorados. $\mathrm{O}$ que, de modo geral, parece ser apenas uma experimentação inocente e excesso temporário, pode resultar em diversos problemas $^{(23)}$.

Dado importante a ser destacado é que houve diferença considerável na 
pontuação média da escala SRSS entre as escolas (escola A: 21 pontos versus escola B: 12,9 pontos).

Diferenças na magnitude dos valores da escala refletem diferenças na percepção que o indivíduo tem do quanto a espiritualidade tem relevância e exerce influência em sua vida ${ }^{(19)}$.

A respeito da afiliação religiosa, a classificação do AUDIT e a escala de espiritualidade houve diferenças significativas. Não ter afiliação religiosa ou ser católico mostrou-se associado ao menor nível de espiritualidade e baixo consumo de álcool. Os estudantes espíritas se subdividiram nos dois níveis de consumo de bebidas alcoólicas e apresentaram altos índices na escala de espiritualidade. Os estudantes evangélicos apresentaram baixo nível de consumo de álcool e baixo nível de espiritualidade (Tabela 5).
Aspectos da religiosidade, tais como: frequentar a igreja, preferência religiosa e importância atribuída à oração e religião apresentaram influência protetora sobre o uso de substâncias em estudantes universitários e adolescentes $^{(18-19)}$.

Há evidências de que a religiosidade exerce controle indireto nas atitudes perante o consumo de substâncias psicoativas, em razão da percepção de que o uso de drogas é um ato moralmente condenável $^{(18-19)}$. Nessa direção, pode-se pensar que os alunos praticantes de alguma religião pertencem a um grupo com valores estabelecidos e normas compartilhadas. Isso explica porque pertencer a uma religião em que há uma condenação mais explícita e clara do uso de álcool, como é o caso dos evangélicos, associa-se ao menor uso de álcool. 


\section{Conclusão}

Este estudo investigou as relações entre fatores de risco, níveis de espiritualidade e uso de álcool em estudantes de dois cursos de enfermagem. O uso do álcool esteve presente em porcentagem considerável de estudantes. Os fatores de risco, associados ao consumo de álcool, foram: pertencer ao sexo masculino, ser casado, ter baixo nível de espiritualidade e não ter afiliação religiosa.

Os resultados obtidos permitiram ampliar conhecimentos sobre a relação entre níveis de espiritualidade e o fenômeno do uso do álcool em estudantes de Enfermagem. A avaliação e monitoramento desse comportamento fornecem informações importantes quanto à magnitude do fenômeno, no âmbito universitário. Estudos sobre o consumo de álcool em estudantes universitários, embora com limitações, viabilizam discussões a respeito do tema. A comparação dos resultados possibilita o planejamento de programas de prevenção mais condizentes com as características e necessidade de cada comunidade, contribuindo para o desenvolvimento de estratégias preventivas e a busca por melhor qualidade de vida.

\section{Referências}

1. Webb E, Ashton H, Kelly P, Kamali F.
Patterns of alcohol consumption, smoking and illicit drug use in British university students: interfaculty comparisons. Drug 
Alcohol Depend. 1997;(47):145-53.

2. Silva Leonardo VER. Malbergier A, Stempliuk VA, Andrade AG. Fatores associados ao consume de álcool e drogas entre estudantes universitários. Rev Saúde Pública. 2006;40(2): 280-8.

3 Pillon SC, Webster, CMC. Teste de identificação de problemas relacionados ao uso de álcool entre estudantes universitários. Rev Enferm UERJ. 2006;14(3):325-32.

4. Hughes SP, Dodder RA. Changing the minimum drinking age: results of a longitudinal study. J Stud Alcohol. 1992;53(6):568-75.

5. Gnadt B. Religiousness, current substance use, and early risk indicators for substance abuse in nursing students. $\mathbf{J}$ Addict Nurs. 2006;17(3):151-8.

6. Rodrigues AP. Souza AS, Zaleski EGF, Arantes SL. Avaliação do nível de propensão para o desenvolvimento de alcoolismo entre estudantes do curso de graduação em enfermagem da Universidade Católica Dom Bosco. SMAD, Rev. Eletrônica Saúde Mental Álcool Drog. (Ed. Port.) Ribeirão Preto, 2007;(3)1. Disponível em http://pepsic.bvsalud.org/scielo.php?scrip $\underline{\mathrm{t}}=$ $\underline{\text { sci } \_ \text {arttext\&pid }=S 1806697620070001000}$ $\underline{05 \& \lg =\mathrm{pt} \& n \mathrm{~nm}=\mathrm{iso}}$. acessos em 21 fev. 2010 .

7. Balan TG, Campos CJG. Padrão de consumo de bebidas alcoólicas entre graduandas de enfermagem de uma universidade estadual paulista. SMAD, Rev. Eletrônica Saúde Mental Álcool Drog. (Ed. Port.) 2006;(2). [acesso 21 fev 2010].

Disponível em http://pepsic.bvsalud.org/scielo.php?scrip $\underline{\mathrm{t}=\mathrm{sci} \text { arttext\&pid=S18066976200600020 }}$ $\underline{0003 \& \operatorname{lng}=p t \& n r m=i s o}$. 
8. Floripes TMF. Beber se embriagando (binge drinking): estudo de uma população de estudantes universitários que fazem uso do álcool de risco. [Dissertação de Mestrado]. Botucatu: Faculdade de Medicina de Botucatu da Universidade Estadual Paulista; 2008.

9. Peuker AC, Fogaça J, Bizarro L. Expectativas e beber problemático entre universitários. Psicol Teoria Pesqui. 2006;22(2):193-200.

10. Michalak L, Trocki K, Bond J. Religion and alcohol in the U. S. National Alcohol Survey: how important is religion for abstention and drinking?

Drug and Alcohol Depend. 2007;(87): $268-80$.

11. Bastos F, Bertoni N, Hacker MA. Consumo de álcool e drogas: principais achados de pesquisa de âmbito nacional, Brasil 2005. Rev Saúde Pública. 2008;42(1):109-17.
12. Prendergast M. Substance Use and Abuse Among College Students: A Review of the Recent Literature. Coll Health. 1994;(43):99-113.

13. Parfrey PS. The effect of religious factors on intoxicant use. Scand J Soc Med. 1976;4(3): 135-40.

14. Lotufo F Neto. Psiquiatria e religião: a prevalência de transtornos mentais entre ministros religiosos. [Tese de LivreDocência]. São Paulo: Faculdade de Medicina da Universidade de São Paulo; 1997.

15. Dantas CR, Pavarin LB, Dalgalarrondo. Sintomas de conteúdo religioso em pacientes psiquiátricos. Rev Bras Psiquiatria. 1999;21(3):58-64.

16. Dalgalarrondo P. Soldera MA, Côrrea-Filho HR. Religião e uso de drogas por adolescentes. Rev Bras Psiquiatria. 2004;26(2):2-90.

17. Piko BF, Fitzpatrick KM. Substance 
use, religiosity, and other protective factors among Hungarian adolescents. Addictive Behav. 2004;(29):1095-107.

18. Galanter M. Dermatis H, Bunt G, Williams C, Trujillo, Steink. Assessment of spirituality and its relevance to addiction treatment. J Substance Abuse Treat. 2007;(33):257-64.

19. Gonçalves MAS, Pillon SC. Adaptação transcultural e avaliação da consistência interna da versão em português da Spirituality Rating Scale. Rev Psiquiatr Clín. 2009;36(1):10-15.

20. National Institute on Alcohol Abuse and Alcoholism (NIAAA). [Internet]. Publications; Alcohol Use Disorders Identification Test (AUDIT). 2000. [acesso 15 abril 2010]. Disponível em: http://www.niaaa.nih.gov/NR/rdonlyres/2
87137A9-62BF-4EDE-A752-

\section{A351C57A0B8/0/Audit.pdf}

21. National Institute on Alcohol Abuse and Alcoholism (NIAAA) (USA). [Internet]. A call to action: Changing the culture of drinking at U.S. Colleges. Bethesda: Task Force on College Drinking, National Institute on Alcohol Abuse and Alcoholism; 2002.

22. Barrias ACR, Queiroz S, Niscatri S, Andrade AG. Comportamento do universitário da área biológica da Universidade de São Paulo em relação ao uso de drogas. Rev Bras Psiquiatria. 2000;27(4):215-24.

23. Edwards G, Marshall EJ, Cook CCH.

O tratamento do alcoolismo: um guia para profissionais de saúde. Porto Alegre: Artes $\quad 2005$. 


\section{Como citar este artigo:}

Pillon SC, Santos SC, Gonçalves AMS, Araújo KM, Funai A. Fatores de risco, níveis de espiritualidade e uso de álcool em estudantes de dois cursos de enfermagem. SMAD, Rev. Eletrônica Saúde Mental Álcool Drog. (Ed. port.) [Internet]. 2010 [acesso: dia mês abreviado com ponto ano]; 6(Especial):493-513. Disponível em: Endereço Eletrônico Visitado. 